\title{
Impact of Pseudomonas aeruginosa quorum sensing on biofilm persistence in an in vivo intraperitoneal foreign-body infection model
}

Correspondence

Thomas Bjarnsholt

tbj@biocentrum.dtu.dk

Received 17 January 2007

Revised 5 March 2007

Accepted 13 March 2007
Louise D. Christensen, ${ }^{1}$ Claus Moser, ${ }^{2}$ Peter Ø. Jensen, ${ }^{2}$

Thomas B. Rasmussen, ${ }^{3}$ Lars Christophersen, ${ }^{2}$ Staffan Kjelleberg, ${ }^{4}$ Naresh Kumar, ${ }^{5}$ Niels Høiby, ${ }^{2}$ Michael Givskov ${ }^{1}$ and Thomas Bjarnsholt ${ }^{1}$

\author{
${ }^{1}$ Bioscience and Technology, BioCentrum, Technical University of Denmark, DK-2800 Lyngby, \\ Denmark \\ ${ }^{2}$ Department of Clinical Microbiology, Rigshospitalet, DK-2100 Copenhagen $\varnothing$, Denmark \\ ${ }^{3}$ Genomics and Strain Development, Chr. Danish Hansen A/S, Bøge Alle 10-12, DK-2970, \\ Hørsholm, Denmark \\ ${ }^{4}$ Center for Marine Biofouling and Bioinnovation, University of New South Wales, NSW 2052, \\ Australia \\ ${ }^{5}$ School of Chemistry, University of New South Wales, NSW 2052, Australia
}

\begin{abstract}
Pseudomonas aeruginosa is an opportunistic human pathogen that causes chronic biofilm-based infections in host organisms. P. aeruginosa employs quorum sensing (OS) to control expression of its virulence, and to establish and maintain chronic infections. Under such conditions, the biofilm mode of growth contributes significantly to $P$. aeruginosa tolerance to the action of the innate and adaptive defence system and numerous antibiotics. In the present study, an in vivo foreign-body infection model was established in the peritoneal cavity of mice. Experimental data showed that QS-deficient $P$. aeruginosa are cleared more rapidly from silicone implants as compared to their wild-type counterparts. Concurrently, treatment with the QS inhibitor furanone C-30 of mice harbouring implants colonized with the wild-type $P$. aeruginosa resulted in a significantly faster clearing of the implants as compared to the placebo-treated group. These results were obtained with both an inbred $(B A L B / c)$ and an outbred (NMRI) mouse strain. The present results support a model by which functional aS systems play a pivotal role in the ability of bacteria to resist clearing by the innate immune system and strongly suggest that the efficiency of the mouse innate defence against biofilm-forming $P$. aeruginosa is improved when the bacteria are treated with QS drugs that induce QS deficiency.
\end{abstract}

\section{INTRODUCTION}

In the USA, microbial infections on medical implants occur in more than 2 million surgical cases each year and infection is the main cause of biomedical implant failure (Emerson \& Camesano, 2004; Gristina, 1987; Verkerke et al., 1997). One explanation gaining momentum is that the biofilm mode of growth protects the colonizing bacteria and enables them to withstand host immune responses and exhibit high tolerance to treatment with the highest deliverable doses of antibiotics (Bjarnsholt et al.,

Abbreviations: AHL, N-acyl-L-homoserine lactone; BAL, bronchoalveolar lavage; C4-HSL, N-butanoyl-L-homoserine lactone; CLSM, confocal laser scanning microscopy; GFP, green fluorescent protein; 3-oxo-C12HSL, N-(3-oxododecanoyl)-L-homoserine lactone; PMN, polymorphonuclear leukocyte; POS, Pseudomonas quinolone signal; OS, quorum sensing. 2005a; Costerton et al., 1999; Hentzer et al., 2003; Høiby et al., 2001; Middleton et al., 2002; Singh et al., 2002). In general, infections on foreign bodies will not be cleared until the implants have been removed from the body (Neut et al., 2005), and unfortunately reimplantation has a high failure rate and is prone to recolonization by similar problem-causing bacteria (Cherney \& Amstutz, 1983).

One particularly notorious biofilm former that is known to cause infections on medically implanted foreign bodies is Pseudomonas aeruginosa (Braxton et al., 2005; Neut et al., 2005). P. aeruginosa uses cell-to-cell communication or quorum sensing (QS) to control the production and secretion of virulence factors, which, deployed at the right time and place, enables evasion of the host defences (Fuqua et al., 1994). By producing and receiving small diffusible $N$ acyl-L-homoserine lactone (AHL) signal molecules, the bacteria are able to monitor their own population size and 
thereby pool their activities accordingly. When the bacterial density, and thus the concentration of secreted signal molecules, reaches a threshold, the signal molecules bind to a transcriptional regulator, forming a complex that induces the transcription of target genes (Fuqua et al., 1994). P. aeruginosa employs two AHL-mediated QS circuits, the las and rhl QS systems. Both systems are organized with a transcriptional regulator, LasR or RhlR, and a synthetase, LasI or RhlI. LasI and RhlI synthesize the two AHL signal molecules $N$-(3-oxododecanoyl)-L-homoserine lactone (3-oxo-C12-HSL) and N-butanoyl-L-homoserine lactone (C4-HSL), respectively (Ochsner \& Reiser, 1995; Passador et al., 1993; Pearson et al., 1994, 1995). The QS systems of $P$. aeruginosa are hierarchically arranged, with the las QS system controlling the $r h l$ QS system (Latifi et al., 1996; Pesci et al., 1997). In addition to the AHL signal molecules produced by $P$. aeruginosa, a third signalling compound, denoted the Pseudomonas quinolone signal (PQS), has been identified. LasR QS has been shown to regulate $\mathrm{PQS}$ production and since $\mathrm{PQS}$ enhances the expression of $r h l I$ and $r h l R$, it is suggested that PQS activity forms a regulatory link between the las and $r h l$ QS systems (Diggle et al., 2003; Pesci et al., 1997). The signal molecule 3-oxo-C12-HSL is known to pose immunomodulatory effects. Thus it was shown to cause neutrophil apoptosis and thereby to facilitate infection (Tateda et al., 2003). Furthermore, 3-oxo-C12-HSL has been shown to inhibit $\mathrm{T}$-cell proliferation and to suppress the production of the cytokine IL-12 and tumour necrosis factor alpha (TNF- $\alpha$ ). These are all involved in the bactericidal $\mathrm{T}_{\mathrm{H}} 1$ response, which is important in the response to bacterial infections (Telford et al., 1998). The PQS signal is also known to exhibit immunomodulatory properties in that it was shown that PQS inhibits T-cell proliferation as 3-oxo-C12-HSL. In contrast to 3-oxo-C12-HSL, PQS induces the production of TNF- $\alpha$ (Hooi et al., 2004). These findings indicate that the signal molecules both promote bacterial communication and production of virulence factors, and suppress the response of the host immune system. This leads to an increased survival potential of the bacteria (Hooi et al., 2004).

P. aeruginosa in which QS is blocked either by mutation or by administration of QS-inhibitory drugs has been shown to be attenuated with respect to virulence (Bjarnsholt et al., 2005a, b; Hentzer et al., 2003; Rasmussen et al., 2005a, b). Davies et al. (1998) showed that biofilms of a P. aeruginosa QS mutant are more susceptible to SDS than the wild-type. This apparent synergistic action with antimicrobial treatment was corroborated by Hentzer et al. (2003), who demonstrated that $P$. aeruginosa PAO1 biofilms became more susceptible to tobramycin and SDS treatments when pretreated with QS inhibitors such as the halofuranones C-30 and C-56. Both compounds have been shown to exhibit QS inhibitory and attenuating effects in vivo; mice with PAO1 lung infections cleared bacteria significantly faster when treated with furanone $\mathrm{C}-30$ or $\mathrm{C}-56$ as compared to the untreated control group (Hentzer et al., 2003; Wu et al.,
2004). A wide range of both natural and chemically synthesized compounds have been tested for QS-inhibitory effects both in vitro and in vivo. In addition to C-30 and C-56, garlic extract, 4-nitropyridine- $\mathrm{N}$-oxide (4-NPO) and patulin are some of the compounds that have been shown to inhibit QS and to significantly reduce $P$. aeruginosa biofilm tolerance to tobramycin in vitro (Rasmussen et al., 2005a, b). Garlic extract and patulin were also demonstrated to promote rapid clearance of $P$. aeruginosa in a pulmonary infectious mouse model (Bjarnsholt et al., 2005b; Rasmussen et al., 2005b). Recently, Bjarnsholt et al. (2005a) also showed that blocking QS, either by mutation or by inhibition with a QS-inhibitory drug, resulted in $P$. aeruginosa biofilms sensitive to treatment with $\mathrm{H}_{2} \mathrm{O}_{2}$ in vitro. Macrolides such as azithromycin have been proposed to inhibit QS (Tateda et al., 2001). However, the concentrations used to determine the effect on QS-controlled gene expression were growth inhibitory. Azithromycin reduces the production of several of the virulence factors of $P$. aeruginosa, including elastase and rhamnolipids. It remains to be demonstrated whether these effects are specifically mediated through the quorum sensors and consequently independent of growth inhibition. Whatever the underlying mechanism is, azithromycin does improve the respiratory function in cystic fibrosis patients (Saiman, 2004; Southern \& Barker, 2004).

Phagocytic cells, such as macrophages and polymorphonuclear leukocytes (PMNs), play an important role in innate immunity as the first line of host defences (Goldsby et al., 2003). When PMNs are exposed to bacteria, they react by phagocytosis and secretion of antimicrobial agents, including oxygen radicals. However, wild-type $P$. aeruginosa biofilms have been shown to impair the activity of PMNs in vitro. Bjarnsholt et al. (2005a) observed that wild-type biofilms survived exposure to freshly isolated PMNs, whereas a $\Delta$ lasR rhlR QS mutant biofilm became penetrated and partly eliminated. There are at least two reasons for this: the wildtype biofilm failed to activate the PMNs to produce $\mathrm{H}_{2} \mathrm{O}_{2}$ and the PMNs appeared paralysed on top of the biofilm bacteria (Bjarnsholt et al., 2005a). In addition, P. aeruginosa wild-type biofilms were able to activate PMNs when they had been grown and developed in the presence of furanone C-30 (Bjarnsholt et al., 2005a). Recently, Jensen et al. (2007) showed that $P$. aeruginosa lyses the PMNs by QS-regulated excretion of rhamnolipid, strongly suggesting that this is a mechanism by which $P$. aeruginosa can evade the host innate immune system. Since rhamnolipid production and hence killing of PMNs was impaired in a QS mutant, we envision that QS-inhibitory drug treatment of $P$. aeruginosa-based biofilm infections would be expected to induce susceptibility to the action of PMNs and thereby promote clearing. In the present investigation we set out to probe this on an in vivo silicone implant model of $P$. aeruginosa infection.

\section{METHODS}

Bacterial strains. The $P$. aeruginosa wild-type strain was obtained from Professor Barbara Iglewski (University of Rochester Medical 
Center, NY, USA). Construction of the corresponding $\Delta$ lasR rhlR mutant was described by Bjarnsholt et al. (2005a). Both strains, wildtype and the $\Delta l a s R$ rhlR mutant, were tagged with a plasmid-based mini-Tn7 transposon system (pBK-miniTn7-gfp3) constitutively expressing a stable green fluorescent protein (GFP) according to Koch et al. (2001).

Preparation of implants. Silicone implants were cut out from a sheet of pyrogen-free silicone with a height of $0.8 \mathrm{~mm}$, in sizes of $5 \times 5 \mathrm{~mm}$. Implants were sterilized in $0.9 \%$ sodium hypochlorite overnight, and washed in sterile distilled water before use. A maximum of 10 implants was placed in $50 \mathrm{ml}$ shake flasks with 20 $\mathrm{ml}$ of cultures of the $P$. aeruginosa wild-type or its isogenic $\Delta$ lasR rhlR mutant. The cultures were prepared by centrifuging $(3000 \boldsymbol{g})$ overnight cultures grown in Luria-Bertani (LB) followed by resuspension in $0.9 \% \mathrm{NaCl}$ to an $\mathrm{OD}_{600}$ of 0.05 (for pre-colonizing the implants destined for BALB/c mice) or 0.5 (for NMRI mice). The implants were incubated in the flasks for $20 \mathrm{~h}$ at $37^{\circ} \mathrm{C}$ and shaking at 110 r.p.m. to get bacterial adhesion.

Animals. Female BALB/c and NMRI mice were purchased from M \& B Laboratory Animals at 9-11 weeks of age. The mice were maintained on standard mouse chow and water ad libitum for a minimum of 1 week before challenge. All animal experiments were authorized by the National Animal Ethics Committee, Denmark.

Challenge protocol. The mice were anaesthetized by subcutaneous injections in the groin area with $0.15 \mathrm{ml}(\mathrm{BALB} / \mathrm{c})$ or $0.25 \mathrm{ml}$ (NMRI) Hypnorm/midazolam (Roche) (2.5 mg Hypnorm ml $\mathrm{mb}^{-1}$ and $1.25 \mathrm{mg}$ midazolam ml $\mathrm{m}^{-1}$ in sterile water). An incision of approximately $1 \mathrm{~cm}$ was made in the groin area, allowing access to the peritoneal cavity, in which the implant was inserted. The incision was sutured with silk and healed without any complications. After insertion of the implants the mice were injected with $1 \mathrm{ml} 0.9 \% \mathrm{NaCl}$ in the neck-skin area to avoid dehydration. Pentobarbital (DAK), $2.0 \mathrm{ml}$ (kg body weight) ${ }^{-1}$, was used to euthanize the mice at termination of the animal experiments (Pedersen et al., 1990).

Bacteriology. After removal from the mice, silicone implants were placed in $5 \mathrm{ml} 0.9 \% \mathrm{NaCl}$ and whirly-mixed for $30 \mathrm{~s}$. The bacteria were then scraped off with a sterile scalpel and whirly-mixed for another $60 \mathrm{~s}$, after which $100 \mu \mathrm{l}$ was serially diluted and plated on blue agar plates (State Serum Institute, Denmark), for bacterial visualization. Blue agar plates are selective for Gram-negative bacilli (Høiby, 1974). The plates were incubated at $37{ }^{\circ} \mathrm{C}$ overnight.

Preparation of the QS inhibitor for injection. The furanone C-30 solution was prepared by dissolving the furanone C-30 powder in $96 \%$ ethanol to a concentration of $5 \mathrm{mg} \mathrm{ml}^{-1}$. This solution was then diluted $1: 40$ in $0.9 \% \mathrm{NaCl}$. The mice were given $1.2 \mu \mathrm{g}$ furanone C-

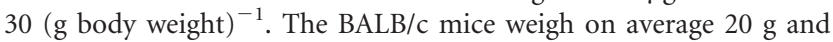
the NMRI mice $30 \mathrm{~g}$, thus $0.2 \mathrm{ml}$ was injected into BALB/c mice and $0.3 \mathrm{ml}$ into NMRI mice. The mice were treated with the inhibitor every $8 \mathrm{~h}$ (intraperitoneal injections) for 4 days starting just after insertion of the implant. Treatment was continued until $24 \mathrm{~h}$ before the mice were euthanized. The placebo group were injected with $0.2 \mathrm{ml}(\mathrm{BALB} / \mathrm{c})$ or $0.3 \mathrm{ml}$ (NMRI) of a $2.4 \%$ ethanol solution $(96 \%$ ethanol in $0.9 \% \mathrm{NaCl})$, corresponding to the amount of ethanol that the furanone C-30-treated group received.

Biofilm development on implants. Biofilm development on the implants was examined using confocal laser scanning microscopy (CLSM) (Zeiss LSM 510 system, Carl Zeiss) equipped with an argon laser for excitation of the fluorophores. Images were obtained with a $40 \times / 0.75$ oil objective. Bacteria were visualized using GFP-tagging, and the IMARIS software package (Bitplane) was used to generate pictures of the biofilms.
Statistical analysis. To compare the data between two groups the Mann-Whitney $U$-test was used (analysis of non-parametric data). $P$ values $\leqslant 0.05$ were considered significant. For calculating $P$-values, the statistical program StatView 5.0.1 (SAS Institute) was used.

\section{RESULTS}

\section{Clearance of $\boldsymbol{P}$. aeruginosa from silicone implants is QS dependent}

The previous findings that QS-deficient $P$. aeruginosa in vitro biofilms are less tolerant to components of the host defence (Bjarnsholt et al., 2005a; Hentzer et al., 2003) led to the hypothesis that mice would be able to clear silicone implants colonized with a QS mutant faster than the wildtype. In order to test this hypothesis, we inserted silicone implants, pre-colonized with either the wild-type or the $\Delta$ lasR rhlR mutant, in the peritoneal cavity of two groups of mice respectively. Thirty-two BALB/c mice were assigned to each of the two groups from which six implants were removed each day at days 4, 7, 14 and 21 post-infection for determination of bacterial counts (c.f.u.) and a further two implants per day were examined by means of CLSM. At the time of implantation, the median c.f.u. for the wild-type and the $\Delta l a s R$ rhlR mutant was adjusted to $7.1 \times 10^{5}$. As shown in Fig. 1(a), the implants colonized with the QS mutant showed a rapid decline with respect to bacterial counts and on day 4 only $1.5 \times 10^{2}$ c.f.u. per implant were found. From day 7 post-infection, no bacteria were found on the implants. In contrast, the bacterial counts on the wild-type-colonized implants decreased more slowly and were, after 4,7 and 14 days, $2.1 \times 10^{4}, 3.4 \times 10^{3}$ and $8.1 \times 10^{2}$, respectively. However, on day 21 no bacteria were found on four out of the six removed implants (Fig. 1a). A significantly lower number of bacteria were present on the implants initially colonized with the $\Delta$ lasR rhlR mutant as compared to the wild-type at days 4,7 and 14 post-infection $(P=0.01 ; P=0.007$ and $P=0.006$, respectively) (Fig. 1a).

Similar experiments were performed with NMRI mice except that the two groups consisted of 28 mice each, and the implants were removed at days $4,7,11$ and 15 postinfection. Five implants were removed from each group at each time point for determination of bacterial counts and two implants per day were examined by CLSM. The c.f.u. per implant for each mouse was determined (Fig. 1b). At the time of implantation the median c.f.u. for the wild-type and the $\Delta$ lasR rhlR mutant were $1.0 \times 10^{7}$ and $8.8 \times 10^{6}$, respectively. Similar to the BALB/c mice, we observed that the c.f.u. for implants colonized with the $\Delta l a s R$ rhlR mutant decreased faster than the wild-type. From day 7 post-infection, no bacteria could be found on the QS mutant pre-colonized implants. The c.f.u. for the wildtype-colonized implants were found to decrease from day 0 to day $4\left(5.6 \times 10^{3}\right)$, thereafter reaching a steady state until day 11 (Fig. 1b). However, on day 15 post-infection, a large increase to $2.2 \times 10^{6}$ c.f.u. per implant was observed 
(a)

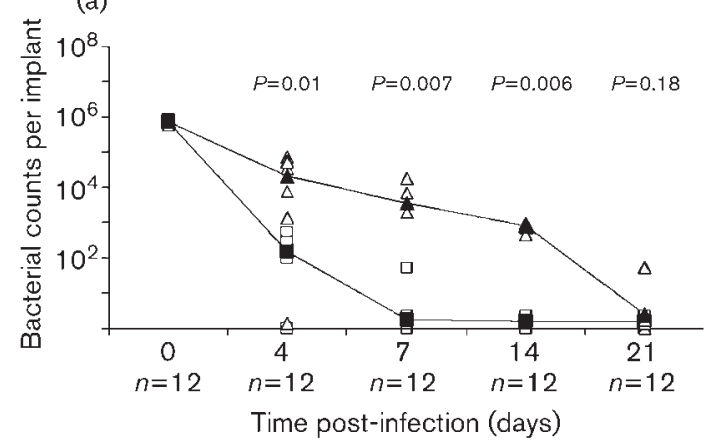

(b)

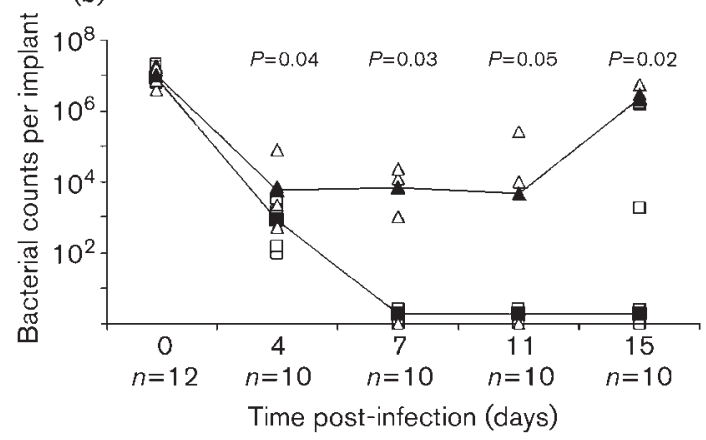

Fig. 1. Implant bacteriology. Healthy BALB/c (a) and NMRI (b) mice had implants pre-colonized with wild-type PAO1 or the $\Delta / a s R$ $r h / R$ mutant inserted in the peritoneal cavity. The number of bacteria adhering to the implants was counted before insertion ( $n=2 \times 6$ implants in both experiments). BALB/c mice: 6 implants from each group were removed on days $4,7,14$ and 21 postinfection $(n=48)$. NMRI mice: 5 implants were removed from each group on days $4,7,11$ and 15 post-infection $(n=40)$. Triangles represent wild-type bacteria, and squares the $\Delta / a s R$ rhIR mutant. Each open triangle and square represents one mouse. Solid black symbols represent the medians for each day. The statistical significance of difference in clearance between the wild-type and $\Delta$ lasR rhIR mutant was tested by a Mann-Whitney $U$-test for nonparametric data. $P$-values are shown in the figure.

(Fig. 1b). Based on the $P$-values, $P=0.04, P=0.03, P=0.05$ and $P=0.02$, respectively, the experiment showed a significant difference in clearance between the wild-typeand QS mutant-colonized implants at all time points (Fig. 1b).

Macroscopic evaluation of the removed implants indicated that the host response against $P$. aeruginosa differed between the two mouse strains. The $\mathrm{BALB} / \mathrm{c}$ mice that were able to clear the biofilms present on the implants showed no macroscopic signs of inflammation. The NMRI mice that were unable to clear the infection showed abscess formation in the tissue surrounding all the wild-type implants at day 15 post-infection. In neither of the mouse strains was pus or abscess formation observed around the $\Delta$ lasR rhlR implants.

\section{CLSM examination of the implants}

After removal from the mice, the implants were inspected by CLSM. The immediate observations were that a significant part of the cellular material adhering to the implants originated from the host and was auto-fluorescent (data not shown). Although this somewhat complicated identification of $P$. aeruginosa biofilms, areas free of mouse tissue were localized, making it possible to obtain images of microcolonies present on the implants removed from BALB/c mice. Fig. 2 shows images obtained from implants removed from $\mathrm{BALB} / \mathrm{c}$ mice on day 4 after insertion. This revealed the presence of microcolony-based biofilms on the implants, though distinctly different from the highly structured and mushroom-shaped biofilms $P$. aeruginosa is known to develop under certain growth conditions in the in vitro flow-cell system (Klausen et al., 2003). Microcolonies were also observed on wild-type-colonized implants removed on days 7 and 14 from BALB/c mice, and on days 7, 11 and 15 from NMRI mice (images not shown). We found a substantially higher number of microcolonies on the implants that were pre-colonized with the wild-type compared to the implants pre-colonized with the $\Delta$ lasR rhlR mutant (Fig. 2). Furthermore, the microcolonies found on the QS mutant-colonized implants were smaller in size. These observations are in agreement with the previous determinations of bacterial content by means of c.f.u. It is also noteworthy that both the $\Delta$ lasR $r h l R$ and wild-type bacteria adhering to the implants were

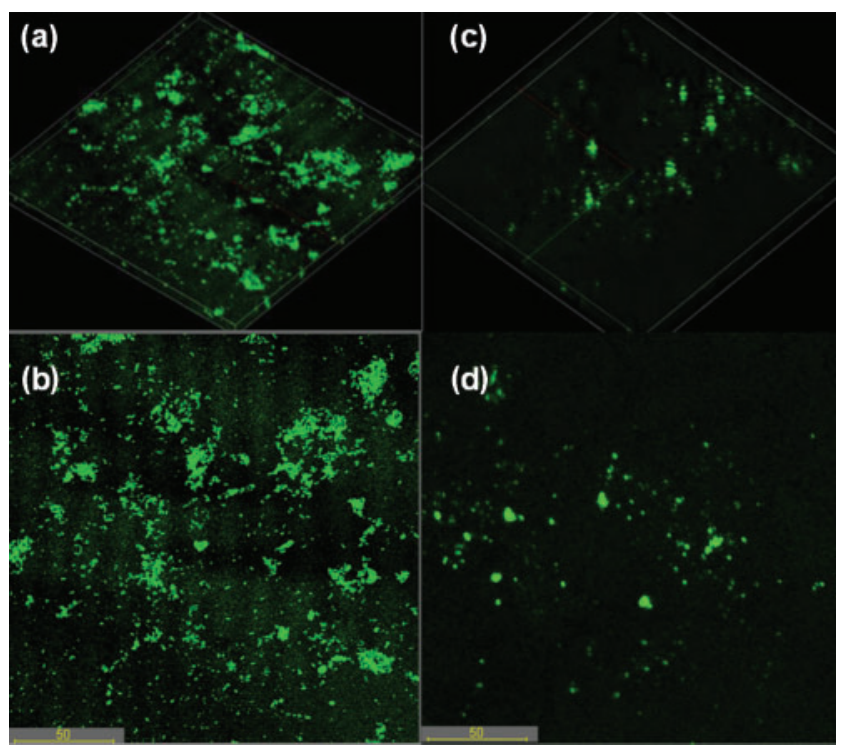

Fig. 2. CLSM images of the wild-type (a, b) and $\Delta / a s R r h / R$ mutant $(c, d)$ colonizing silicone implants after removal from BALB/ $c$ mice at day 4 after insertion. Green fluorescent areas represent GFP-tagged microcolonies. (a, c) 3D images of the microcolonies adhering to the implants; (b, d) top views. Scale bars $(50 \mu \mathrm{m})$ are shown in (b) and (d). All images were obtained with a $40 \times / 0.75$ oil objective. 
not rod-shaped, as at the time of implantation, but rather sphere-shaped and smaller than exponentially growing, batch-cultured cells (Fig. 3).

\section{Furanone C-30 treatment of wild-type $P$. aeruginosa-colonized implants}

Since the QS mutant was cleared faster than the wild-type bacteria, administration of a functional QS-inhibitory drug would be expected to have a similar impact. Forty NMRI mice had implants pre-colonized with wild-type $P$. aeruginosa $\mathrm{PAO} 1\left(\mathrm{OD}_{600}\right.$ 0.5) inserted in the peritoneal cavity at day 0 . Immediately after this, the mice were separated into two groups (20 in each), which received intraperitoneal injections of either furanone C-30 $[\sim 1 \mu \mathrm{g}$ (g body weight $)^{-1}$ ] or placebo with $8 \mathrm{~h}$ intervals for the following 4 days. This concentration of drug had previously been shown to significantly improve clearance of $P$. aeruginosa from a pulmonary model of infection (Hentzer et al., 2003). No macroscopic effect on the host was observed when treating the mice with furanone C-30. Twenty-four hours after the last injection, the implants were removed at day 5. This protocol was repeated in two individual experiments [denoted (a) and (b)] in which the c.f.u. were adjusted to $8.2 \times 10^{6}$ per implant. Determination of c.f.u. was used to assess the effect of furanone C-30 treatment on clearance, and the c.f.u. per implant for each mouse in each group was determined and the medians plotted in Fig. 4(a, b). The remaining implants were used for CLSM studies. In experiment (a), the c.f.u. determined for the treatment and placebo groups showed medians of 800 and $2.6 \times 10^{6}$, respectively $(P=0.005)$. In

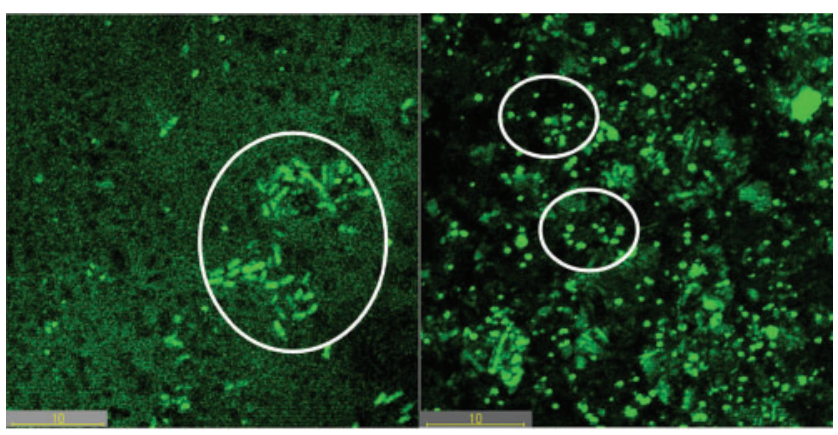

Fig. 3. CLSM images of implants colonized with wild-type $P$. aeruginosa. The image to the left was taken after colonization but before insertion, while the image to the right is of an implant removed 15 days after insertion from a NMRI mouse. It is noteworthy that both the $\Delta / a s R$ rhIR and wild-type bacteria adhering to the implants, after removal from the mice, were not rod-shaped, as at the time of implantation, but rather sphereshaped and smaller than exponentially growing, batch-cultured cells. This phenomenon was observed when the implants were removed 7 and 15 days after insertion $(n=8)$. Scale bars $(10 \mu \mathrm{m})$ are shown on the images. The images were obtained with a $40 \times 1$ 0.75 oil objective and then magnified five times. (a)

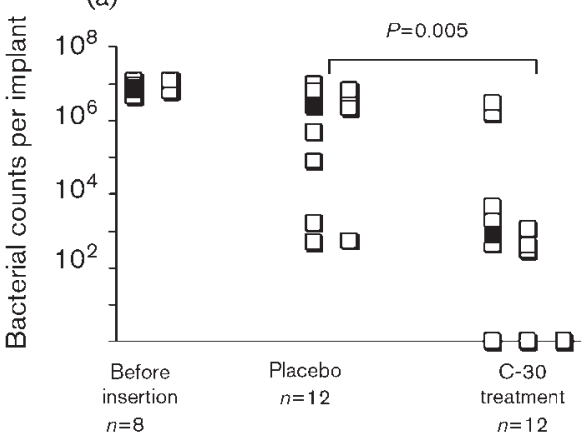

(b)

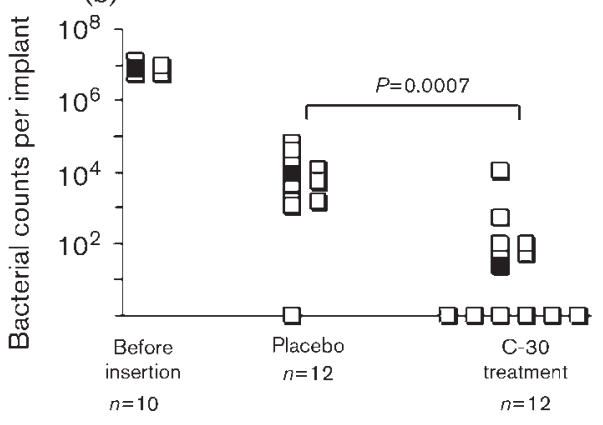

Fig. 4. Implant bacteriology. Healthy NMRI mice had implants precolonized with wild-type PAO1 inserted in the peritoneal cavity. (a) and (b) are two individual experiments carried out identically with NMRI mice, where 24 implants were used in each study for bacteriology. The mice were in each experiment separated into two groups and were injected with either furanone C-30 $[\sim 1 \mu \mathrm{g}$ (g body weight $)^{-1}$ ] or placebo at $8 \mathrm{~h}$ intervals for 4 days. On day 5 post-infection the implants were removed and the number of bacteria adhering to the implant was counted. Each mouse is represented by one square, and solid squares represent the median of the bacterial counts for each group. Bacterial counts per implant were calculated before insertion [(a) $n=8$ and (b) $n=12]$. The statistical significance of difference in clearance between the furanone C-30-treated and placebo groups was tested by a Mann-Whitney $U$-test for non-parametric data. $P$-values for the experiments are shown in the figure.

experiment (b), the median c.f.u. were 25 (furanone C-30treated) and $8.8 \times 10^{3}$ (placebo) $(P=0.0007)$.

A similar experiment was performed with $\mathrm{BALB} / \mathrm{c}$ mice, where the furanone C-30 treatment also was found to promote clearing of the bacteria from the implants compared to the placebo-treated group $(P=0.03)$ (data not shown).

\section{CLSM examination of the implants}

CLSM was used to study the removed implants. Due to the low bacterial counts on the implants from the furanone C30-treated NMRI mice in experiment (b), no images of bacteria could be obtained. However, bacteria organized in microcolonies were found on several occasions on the 
implants from the placebo groups from experiments (a) and (b), and also some bacteria were observed on the furanone C-30-treated implants from experiment (a) (data not shown). With BALB/c mice, it was also possible to find microcolonies on the implants removed from both the furanone $\mathrm{C}$-30-treated and untreated $\mathrm{BALB} / \mathrm{c}$ mice. A higher number of microcolonies was observed on the implants from the untreated mice as compared to those treated with furanone C-30.

\section{DISCUSSION}

Many researchers share the view that biofilms are sanctuaries in a hostile environment, which release planktonic cells that may spark systemic infections. Numerous studies of in vitro biofilms have revealed a characteristic series of events, which include surface attachment followed by growth and proliferation into a characteristic self-made, exopolymeric embedded state where the bacteria show an extreme tolerance to antimicrobial measures. Microscopic investigations have revealed that biofilms develop commonly on inert surfaces of medical devices or on dead tissue, but they can also form on living tissues. When it comes to in-depth studies, the preferred model organism is undoubtedly $P$. aeruginosa. The genus Pseudomonas is one of the most diverse and ecologically significant groups of bacteria known. $P$. aeruginosa is also the most common bacterium found in nosocomial and life-threatening infections of immune compromised patients (Van Delden \& Iglewski, 1998). As the understandings of pathogenicity, biofilm development and QS as well as the elucidation of the molecular mechanisms behind QS have gained momentum, researchers have conceived several strategies to block QS. Hospitalized patients acquire infections in connection with the use of medical devices such as arteriovenous shunts, pacemakers, urinary and other types of catheters, orthopaedic devices and mechanical heart valves. The biofilm lifestyle is believed to dominate in the above-mentioned chronic bacterial infections. Consequently, they share similar characteristics: they resist the activities of the host immune system and cannot be eradicated with antibiotic intervention regimes. New anti-biofilm measures must therefore be identified which efficiently decrease the biofilm-forming capability or persistence of the infectious bacteria. The central role of QS in virulence and biofilm development has made it easy (at least in academia) to see a direct application for QS-inhibitory drugs.

In vitro biofilms of $P$. aeruginosa in which the $\mathrm{QS}$ communication systems have been disabled by mutation are more prone to killing by tobramycin and hydrogen peroxide (Bjarnsholt et al., 2005a), as well as ciprofloxacin, ceftizidime and kanamycin treatment (our unpublished results), shear forces, protozoan grazing and neutrophil phagocytosis, than biofilms formed by a wild-type counterpart (Allesen-Holm et al., 2006; Bjarnsholt et al., 2005b; Davies et al., 1998; Hassett et al., 1999; Hentzer et al., 2002,
2003; Matz et al., 2004; Rasmussen et al., 2005a, b). Moreover, in animal models these mutants appear attenuated and are more readily cleared by the host organism (Bjarnsholt et al., 2005a, b).

We have developed yet another animal model to study the effect of functional QS on biofilm susceptibility in vivo. By inserting implants pre-colonized with either the $\Delta l a s R$ rhlR mutant or wild-type $P$. aeruginosa in the peritoneal cavity of mice it was shown that the former was cleared significantly faster than the latter from the implants both in BALB/c and NMRI mice. In contrast to the NMRI mice, $\mathrm{BALB} / \mathrm{c}$ mice were eventually able to clear the biofilms present on the $P$. aeruginosa wild-type-colonized implants (Fig. 1). The pathophysiological mechanisms resulting in the different outcomes in the two mouse strains were not the focus of the present study, and will be investigated further in the future.

Buret et al. (1991) performed a foreign-body insertion experiment on rabbits, where silastic implants colonized with $P$. aeruginosa were inserted intraperitoneally. Their results showed a similar pattern to the one observed with the NMRI mice in the present study. In their study, bacterial counts decreased 13 -fold from day 0 to day 4 , but increased 20-fold in bacterial counts from day 4 to day 42 (Buret et al., 1991). Likewise in the study performed by Buret et al. (1991), it was in one case observed that wallingoff and necrosis of host and pathogen debris resulted in abscess formation, which was also observed in the present study upon removal of the wild-type-colonized implants from NMRI mice 15 days post-infection. The bacterial counts were also significantly higher than in cases where no abscess was seen. Due to the abscess formation and high number of bacteria adhering to the implants upon removal, it is likely that the P. aeruginosa wild-type was able to withstand the host immune system in the NMRI mice and establish a successful chronic infection, which was not the case in BALB/c mice.

Similar to the present study, Buret et al. (1991) found host cells attached to the implants. These cells were dominated by PMNs, but erythrocytes and macrophages were also observed (Buret et al., 1991). When removing the implants from the mice it was observed that the bacterial cells had gained an altered morphology, as compared to the rod shape they possessed at insertion. This is a well-known phenomenon observed when Gram-negative cells such as $P$. aeruginosa adapt to environments which provide conditions that sustain only slow or no growth (Clegg et al., 1996; Givskov et al., 1994; Lappin-Scott et al., 1988). Since this phenomenon was observed with both the QS mutant and wild-type strain, change in morphology does not seem to be the reason for the slower clearing of wildtype bacteria from the implants as compared to the mutant.

NMRI and BALB/c mice carrying wild-type-colonized implants were treated with furanone $\mathrm{C}-30$ given as intraperitoneal injections. Two individual experiments 
with NMRI mice and one experiment with $\mathrm{BALB} / \mathrm{c}$ mice were carried out, and it was shown that the furanone C-30treated groups had a significantly lower amount of bacteria adhering to the implants when they were removed from the mice, as compared to the placebo-treated groups. This confirms that treatment of furanone C-30 induces antimicrobial conditions leading to bacterial clearing, as previously shown in an in vivo pulmonary infection model (Hentzer et al., 2003).

It is likely that the difference in clearance observed in the present study is due to the $P$. aeruginosa wild-type elimination of the PMNs and we have recently found that the causative agent is rhamnolipid (Jensen et al., 2007). Interestingly, we found that an ethyl acetate extract of the bronchoalveolar lavage (BAL) fluid of $P$. aeruginosa-infected cystic fibrosis patients was capable of lysing freshly isolated PMNs. Furthermore, it has been shown that treatment with furanone C-30 of a growing batch culture of the $P$. aeruginosa wild-type resulted in blocking of the development of PMN necrotic and haemolytic effects (Jensen et al., 2007). In a recent study of the geneexpression profile of $P$. aeruginosa exposed to freshly isolated PMNs, several QS-regulated genes, which contribute to virulence and immune modulation, were further upregulated in the presence of PMNs (M. Alhede and others, unpublished). Among these were the genes encoding PQS, pyocyanin, rhamnolipid and lectin production, a result also found by Hentzer et al. (2003). Previous analysis has shown that expression of those genes was inhibited by furanone C-30. Taken together, these data strongly suggest that the ability of $P$. aeruginosa to kill the PMNs has been impaired in the furanone C-30-treated groups, thereby resulting in a faster clearance of the bacteria on the implants in both BALB/c and NMRI mice. This is in favour of a model in which administration of furanone C-30 could possibly increase the survival potential of the PMNs, by concomitantly reducing expression of the $P$. aeruginosa PMN-devastating products. Furthermore, furanone C-30 treatment was shown to have an impact on the bacterial clearance in both an inbred and an outbred mouse strain, which is of great advantage. This implies that the treatment effect of the compound is independent of the mouse strain, and hence it offers a guarded optimism that QS inhibitors can play a role in the treatment of various biofilm-based infections in humans.

In the present investigation, the biofilms that were allowed to develop on the silicone implants were formed in $20 \mathrm{~h}$, and thus were very young. Despite this relatively young state, a transcriptomic analysis by Hentzer et al. (2005) on in vitro biofilms showed that the majority of QS-controlled genes were already switched on. Furthermore, this condition is likely to resemble a foreign-body infection in its early stages. In the future we aim to investigate mature and fully developed biofilms adhering to the implants. This condition would be expected to be medically relevant for foreign bodies that have been colonized for a longer period due to the fact that biofilm-growing bacteria are able to withstand the host immune system and exposure to most antibiotics (Bjarnsholt et al., 2005a; Costerton et al., 1999; Hentzer et al., 2003; Høiby et al., 2001; Middleton et al., 2002; Singh et al., 2002).

In conclusion, the present in vivo model showed that functional QS plays a key role in the ability of mice to clear a $P$. aeruginosa foreign-body infection. The results indicate that the antimicrobial efficacy of the defence mechanisms to biofilm-growing $P$. aeruginosa on foreign bodies is improved when the bacteria are treated with QS-inhibitory drugs. Accordingly, the present investigation supports a model in which $P$. aeruginosa employs QS-regulated gene expression to circumvent the host response during the initial stages of infection. This model will be suitable for testing and calibrating the concentration of future pharmaceutically relevant QS-inhibitory drugs aiming at the prevention or treatment of $P$. aeruginosa biofilm infections.

\section{ACKNOWLEDGEMENTS}

M. G. received financial support from the Danish Research Council FTP supported 'A new approach to the control of microbial activity', the Biomedical Consortium 'Biomed', Mölnlycke Health Care, AB Wound Care R\&D, Sweden, and the German Mukoviszidose e.v. T. B. received financial support from The Carlsberg Foundation and Lundbeck Foundation 'The implication of bacterial biofilms in chronic infections'.

\section{REFERENCES}

Allesen-Holm, M., Barken, K. B., Yang, L., Klausen, M., Webb, J. S., Kjelleberg, S., Molin, S., Givskov, M. \& Tolker-Nielsen, T. (2006). A characterization of DNA release in Pseudomonas aeruginosa cultures and biofilms. Mol Microbiol 59, 1114-1128.

Bjarnsholt, T., Jensen, P. O., Burmolle, M., Hentzer, M., Haagensen, J. A., Hougen, H. P., Calum, H., Madsen, K. G., Moser, C. \& other authors (2005a). Pseudomonas aeruginosa tolerance to tobramycin, hydrogen peroxide and polymorphonuclear leukocytes is quorumsensing dependent. Microbiology 151, 373-383.

Bjarnsholt, T., Jensen, P. O., Rasmussen, T. B., Christophersen, L., Calum, H., Hentzer, M., Hougen, H. P., Rygaard, J., Moser, C. \& other authors (2005b). Garlic blocks quorum sensing and promotes rapid clearing of pulmonary Pseudomonas aeruginosa infections. Microbiology 151, 3873-3880.

Braxton, E. E., Jr, Ehrlich, G. D., Hall-Stoodley, L., Stoodley, P., Veeh, R., Fux, C., Hu, F. Z., Quigley, M. \& Post, J. C. (2005). Role of biofilms in neurosurgical device-related infections. Neurosurg Rev 28, 249-255.

Buret, A., Ward, K. H., Olson, M. E. \& Costerton, J. W. (1991). An in vivo model to study the pathobiology of infectious biofilms on biomaterial surfaces. J Biomed Mater Res 25, 865-874.

Cherney, D. L. \& Amstutz, H. C. (1983). Total hip replacement in the previously septic hip. J Bone Joint Surg Am 65, 1256-1265.

Clegg, C. D., van Elsas, J. D., Anderson, J. M. \& Lappin-Scott, H. M. (1996). Survival of parental and genetically modified derivatives of a soil isolated Pseudomonas fluorescens under nutrient-limiting conditions. J Appl Bacteriol 81, 19-26.

Costerton, J. W., Stewart, P. S. \& Greenberg, E. P. (1999). Bacterial biofilms: a common cause of persistent infections. Science 284, 1318-1322. 
Davies, D. G., Parsek, M. R., Pearson, J. P., Iglewski, B. H., Costerton, J. W. \& Greenberg, E. P. (1998). The involvement of cell-to-cell signals in the development of a bacterial biofilm. Science 280, 295-298.

Diggle, S. P., Winzer, K., Chhabra, S. R., Worrall, K. E., Cámara, M. \& Williams, P. (2003). The Pseudomonas aeruginosa quinolone signal molecule overcomes the cell density-dependency of the quorum sensing hierarchy, regulates rhl-dependent genes at the onset of stationary phase and can be produced in the absence of LasR. Mol Microbiol 50, 29-43.

Emerson, R. J. \& Camesano, T. A. (2004). Nanoscale investigation of pathogenic microbial adhesion to a biomaterial. Appl Environ Microbiol 70, 6012-6022.

Fuqua, W. C., Winans, S. C. \& Greenberg, E. P. (1994). Quorum sensing in bacteria: the LuxR-LuxI family of cell density-responsive transcriptional regulators. J Bacteriol 176, 269-275.

Givskov, M., Eberl, L., Moller, S., Poulsen, L. K. \& Molin, S. (1994). Responses to nutrient starvation in Pseudomonas putida KT2442: analysis of general cross-protection, cell shape, and macromolecular content. J Bacteriol 176, 7-14.

Goldsby, R. A., Osborne, B. A., Kindt, T. J. \& Kuby, J. (2003). Immunology, 5th edn. New York: W.H. Freeman.

Gristina, A. G. (1987). Biomaterial-centered infection: microbial adhesion versus tissue integration. Science 237, 1588-1595.

Hassett, D. J., Ma, J. F., Elkins, J. G., McDermoot, T. R., Ochsner, U. A., West, S. E., Huang, C. T., Fredericks, J., Burnett, S. \& other authors (1999). Quorum sensing in Pseudomonas aeruginosa controls expression of catalase and superoxide dismutase genes and mediates biofilm susceptibility to hydrogen peroxide. Mol Microbiol 34, 1082-1093.

Hentzer, M., Riedel, K., Rasmussen, T. B., Heydorn, A., Andersen, J. B., Parsek, M. R., Rice, S. A., Eberl, L., Molin, S. \& other authors (2002). Inhibition of quorum sensing in Pseudomonas aeruginosa biofilm bacteria by a halogenated furanone compound. Microbiology 148, 87-102.

Hentzer, M., Wu, H., Andersen, J. B., Riedel, K., Rasmussen, T. B., Bagge, N., Kumar, N., Schembri, M. A., Song, Z. \& other authors (2003). Attenuation of Pseudomonas aeruginosa virulence by quorum sensing inhibitors. EMBO J 22, 3803-3815.

Hentzer, M., Eberl, L. \& Givskov, M. (2005). Transcriptome analysis of Pseudomonas aeruginosa biofilm development: anaerobic respiration and iron limitation. Biofilms 2, 37-61.

Høiby, N. (1974). Epidemiological investigations of the respiratory tract bacteriology in patients with cystic fibrosis. Acta Pathol Microbiol Scand [B] Microbiol Immunol 82, 541-550.

Høiby, N., Krogh Johansen, H., Moser, C., Song, Z., Ciofu, O. \& Kharazmi, A. (2001). Pseudomonas aeruginosa and the in vitro and in vivo biofilm mode of growth. Microbes Infect 3, 23-35.

Hooi, D. S. W., Bycroft, B. W., Chhabra, S. R., Williams, P. \& Pritchard, D. I. (2004). Differential immune modulatory activity of Pseudomonas aeruginosa quorum-sensing signal molecules. Infect Immun 72, 6463-6470.

Jensen, P. Ø., Bjarnsholt, T., Phipps, R., Rasmussen, T. B., Calum, H., Christophersen, L., Moser, C., Williams, P., Pressler, T. \& other authors (2007). Rapid necrotic killing of polymorphonuclear leukocytes is caused by quorum-sensing-controlled production of rhamnolipid by Pseudomonas aeruginosa. Microbiology 153, 1329-1338.

Klausen, M., Heydorn, A., Ragas, P., Lambertsen, L., AaesJorgensen, A., Molin, S. \& Tolker-Nielsen, T. (2003). Biofilm formation by Pseudomonas aeruginosa wild type, flagella and type IV pili mutants. Mol Microbiol 48, 1511-1524.

Koch, B., Jensen, L. E. \& Nybroe, O. (2001). A panel of Tn7-based vectors for insertion of the $g f p$ marker gene or for delivery of cloned
DNA into Gram-negative bacteria at a neutral chromosomal site. J Microbiol Methods 45, 187-195.

Lappin-Scott, H. M., Cusack, F., MacLeod, A. \& Costerton, J. W. (1988). Starvation and nutrient resuscitation of Klebsiella pneumoniae isolated from oil well waters. J Appl Bacteriol 64, 541-549.

Latifi, A., Foglino, M., Tanaka, K., Williams, P. \& Lazdunski, A. (1996). A hierarchical quorum-sensing cascade in Pseudomonas aeruginosa links the transcriptional activators LasR and RhIR (VsmR) to expression of the stationary-phase sigma factor RpoS. Mol Microbiol 21, 1137-1146.

Matz, C., Bergfeld, T., Rice, S. A. \& Kjelleberg, S. (2004). Microcolonies, quorum sensing and cytotoxicity determine the survival of Pseudomonas aeruginosa biofilms exposed to protozoan grazing. Environ Microbiol 6, 218-226.

Middleton, B., Rodgers, H. C., Camara, M., Knox, A. J., Williams, P. \& Hardman, A. (2002). Direct detection of $N$-acylhomoserine lactones in cystic fibrosis sputum. FEMS Microbiol Lett 207, 1-7.

Neut, D., Hendriks, J. G., van Horn, J. R., van der Mei, H. C. \& Busscher, H. J. (2005). Pseudomonas aeruginosa biofilm formation and slime excretion on antibiotic-loaded bone cement. Acta Orthop 76, 109-114.

Ochsner, U. A. \& Reiser, J. (1995). Autoinducer-mediated regulation of rhamnolipid biosurfactant synthesis in Pseudomonas aeruginosa. Proc Natl Acad Sci U S A 92, 6424-6428.

Passador, L., Cook, J. M., Gambello, M. J., Rust, L. \& Iglewski, B. H. (1993). Expression of Pseudomonas aeruginosa virulence genes requires cell-to-cell communication. Science 260, 1127-1130.

Pearson, J. P., Gray, K. M., Passador, L., Tucker, K. D., Eberhard, A., Iglewski, B. H. \& Greenberg, E. P. (1994). Structure of the autoinducer required for expression of Pseudomonas aeruginosa virulence genes. Proc Natl Acad Sci U S A 91, 197-201.

Pearson, J. P., Passador, L., Iglewski, B. H. \& Greenberg, E. P. (1995). A second $N$-acylhomoserine lactone signal produced by Pseudomonas aeruginosa. Proc Natl Acad Sci U S A 92, 1490-1494.

Pedersen, S. S., Shand, G. H., Hansen, B. L. \& Hansen, G. N. (1990). Induction of experimental chronic Pseudomonas aeruginosa lung infection with $P$. aeruginosa entrapped in alginate microspheres. APMIS 98, 203-211.

Pesci, E. C., Pearson, J. P., Seed, P. C. \& Iglewski, B. H. (1997). Regulation of las and $r h l$ quorum sensing in Pseudomonas aeruginosa. J Bacteriol 179, 3127-3132.

Rasmussen, T. B., Bjarnsholt, T., Skindersoe, M. E., Hentzer, M., Kristoffersen, P., Kote, M., Nielsen, J., Eberl, L. \& Givskov, M. (2005a). Screening for quorum-sensing inhibitors (QSI) by use of a novel genetic system, the QSI selector. J Bacteriol 187, 1799-1814.

Rasmussen, T. B., Skindersoe, M. E., Bjarnsholt, T., Phipps, R. K., Christensen, K. B., Jensen, P. O., Andersen, J. B., Koch, B., Larsen, T. O. \& other authors (2005b). Identity and effects of quorum-sensing inhibitors produced by Penicillium species. Microbiology 151, 1325-1340.

Saiman, L. (2004). The use of macrolide antibiotics in patients with cystic fibrosis. Curr Opin Pulm Med 10, 515-523.

Singh, P. K., Parsek, M. R., Greenberg, E. P. \& Welsh, M. J. (2002). A component of innate immunity prevents bacterial biofilm development. Nature 417, 552-555.

Southern, K. W. \& Barker, P. M. (2004). Azithromycin for cystic fibrosis. Eur Respir J 24, 834-838.

Tateda, K., Comte, R., Pechere, J. C., Kohler, T., Yamaguchi, K. \& van Delden, C. (2001). Azithromycin inhibits quorum sensing in Pseudomonas aeruginosa. Antimicrob Agents Chemother 45, 19301933. 
Tateda, K., Ishii, Y., Horikawa, M., Matsumoto, T., Miyairi, S., Pechere, J. C., Standiford, T. J., Ishiguro, M. \& Yamaguchi, K. (2003). The Pseudomonas aeruginosa autoinducer $N$-3-oxododecanoyl homoserine lactone accelerates apoptosis in macrophages and neutrophils. Infect Immun 71, 5785-5793.

Telford, G., Wheeler, D., Williams, P., Tomkins, P. T., Appleby, P., Sewell, H., Stewart, G. S., Bycroft, B. W. \& Pritchard, D. I. (1998). The Pseudomonas aeruginosa quorum-sensing signal molecule $\mathrm{N}$-(3oxododecanoyl)-L-homoserine lactone has immunomodulatory activity. Infect Immun 66, 36-42.

Van Delden, C. \& Iglewski, B. H. (1998). Cell-to-cell signaling and Pseudomonas aeruginosa infections. Emerg Infect Dis 4, 551-560.
Verkerke, G. J., Schraffordt Koops, H., Veth, R. P., van Horn, J. R., Postma, L. \& Grootenboer, H. J. (1997). First clinical experience with a noninvasively extendable endoprosthesis: a limb-saving procedure in children suffering from a malignant bone tumor. Artif Organs 21, 413-417.

Wu, H., Song, Z., Hentzer, M., Andersen, J. B., Molin, S., Givskov, M. \& Hoiby, N. (2004). Synthetic furanones inhibit quorum-sensing and enhance bacterial clearance in Pseudomonas aeruginosa lung infection in mice. J Antimicrob Chemother 53, 1054-1061.

Edited by: P. Cornelis 